\title{
The rationale for selenium supplementation in patients with autoimmune thyroiditis, according to the current state of knowledge
}

\author{
Dorota Filipowicz ${ }^{\circledR}$, Karolina Majewska ${ }^{\circledR}$, Anastasia Kalantarova ${ }^{\circledR}$, Ewelina Szczepanek-Parulska ${ }^{\circledR}$, \\ Marek Ruchała ${ }^{(1)}$
}

Department of Endocrinology, Metabolism and Internal Medicine, Poznan University of Medical Sciences, Poznan, Poland

\begin{abstract}
Selenium (Se) supplements are commonly prescribed to autoimmune thyroiditis (AIT) patients by European endocrinologists, despite the lack of official guidelines. The majority of Europe is depleted of natural Se sources, and the daily population intake does not comply with recommended values. Optimal individual plasma Se concentration is reached when the selenoproteins (selenoprotein P, glutathione peroxidase) are fully saturated. However, Se intake has to be regulated because both Se shortage and overdose negatively impact health. In the case of AIT, Se may alleviate symptoms or prevent progression to hypothyroidism and postpartum hypothyroidism. Se supplementation in euthyroid, subclinical, or overt hypothyroid AIT patients decreased thyroid autoantibodies, lowered or maintained the TSH level, decreased the fT4/fT3 ratio, reduced the body's oxidative stress and inflammatory status, and amended quality of life and thyroid ultrasound structure and volume. In pregnant females, adequate Se intake protected them against miscarriages, preeclampsia/hypertension, preterm birth, small-for-gestational-age infants' birth, and improved child's neuropsychological development. In the elderly population, adequate Se supplementation decreased cardiovascular diseases and hypertension risk, but prolonged intake of excessive doses increased the all-cause mortality rate. Routine Se supplementation implementation requires from researchers and clinicians consideration of specific populational differences in natural Se and iodine supply, the patient's clinical situation (supplementation simultaneously or before levothyroxine treatment, AIT/non-AIT hypothyroidism), individual response to supplementation (Se and selenoprotein P assessment), predisposition (genetic testing), the status of other trace elements, and the interplay between those micronutrients. Moreover, the safety of commercially available Se formulations, doses, and duration of treatment should be determined. Proper guidelines are warranted to standardise the medical approach to Se supplementation. This article presents a comprehensive review of recent randomised-controlled trials, meta-analyses, and clinical trials concerning the risks and benefits of Se supplementation in different clinical settings and specific populations with particular emphasis on AIT in a practical manner. (Endokrynol Pol 2021; 72 (2): 153-162)
\end{abstract}

Key words: selenium; thyroid; autoimmune thyroiditis; diet supplements; pregnancy

\section{Introduction}

The influence of diet supplements on autoimmune thyroid disease (AIT) has been debated recently. Growing patients' interest in nutraceutical products as an alternative form of AIT treatment, wide availability and uncontrolled intake of supplements (over-the-counter in most countries) forces scientists and clinicians to expand and update the evidence-based state of knowledge. Although no positive recommendation for selenium (Se) supplementation in AIT has been provided by European or American Endocrine/Thyroid Societies, it is widely supported worldwide in daily clinical practice. According to an Associazione Medici Endocrinologi (AME) Survey completed by 815 doctors (91.6\% endocrinologists) in 2016, the majority of doctors
(79.4\%) prescribe Se to euthyroid AIT patients, aiming for hypothyroidism delay or a decrease of thyroid antibodies (TA) titre. Interestingly, only $20 \%$ of doctors would advocate against Se if a patient requests any form of treatment instead of observation. In patients with subclinical hypothyroidism (SHT) and positive TA, two-thirds of respondents would prescribe $\mathrm{Se}$, whereas only one third - with negative TA, regardless of additional levothyroxine (LT4) treatment. In euthyroid AIT women who are pregnant or conceiving, $40 \%$ of doctors decided on Se supplementation, intending to prevent postpartum thyroiditis (PPT). The preferable dose in all groups was 100-200 $\mu \mathrm{g} /$ day [1]. Surprising results of the previous survey encouraged investigators to expand the questionnaire within the European Thyroid Association (ETA) members (147 physicians, 
$84 \%$ endocrinologists). Sixty-six per cent of them would use Se in AIT; however, only $20 \%$ recognised convincing evidence favouring Se supplementation. In the LT4-treated AIT group, $50 \%$ of responders would prescribe Se, where $71 \%$ would not recommend it in pregnancy. In AIT subjects not receiving LT4 physicians were more likely to support Se supplementation (69\%), and a lower rate $(67 \%)$ would not prescribe it in case of pregnancy. Besides the goals mentioned above, some respondents consider the quality of life (QoL) and thyroid ultrasound (US) image improvement as expected supplementation outcomes. The preferable treatment pattern was $\leq 200 \mu \mathrm{g} /$ day of selenomethionine (SeMet) for weeks to months [2].

This article provides a comprehensive review of the potential role and usefulness of Se supplementation in various clinical settings based on recent randomised-controlled trials (RCTs), meta-analyses, and clinical trials and systemises knowledge concerning the role of Se in human health among different populations, with particular emphasis on AIT, in a practical aspect.

\section{Natural sources and populations' supplies of Se}

Populations' replenishment of Se is country-specific and largely depends on soil content and bioavailability to enter the food chain through plants. The vast majority of the Earth's rocks have a low Se content, but some sedimentary and volcanic rocks are enriched with this trace element, explaining the geographical differences [3]. The majority of Se in our body is obtained from consuming seafood (e.g. tuna, halibut, sardines, shrimps), animal organs and meat (e.g. ham, beefsteak, turkey, beef liver, chicken), cereals, grains and crops (e.g. pasta, brown rice, whole-wheat bread), milk or dairy products (e.g. cottage cheese, milk, eggs), and fruit/vegetables (e.g. spinach, bananas) [4]. However, the food with the highest Se content is Brazil nuts [5]. Importantly, its concentration in nuts is notably diversified depending on the place of origin and brand; it is non-standardized (no product information about Se concentration or geographic region of import) and variable (high seed to seed and batch variation). Analysis of 13 commercially available Brazil nut brands, only from Brazil, showed that the batch from the largest nut producer in the Se-rich Amazonas state exceeded by up to 3.5 times the tolerable upper intake level (UL) for Se in a standard serving of 6-8 nuts. Therefore, the authors opted to reduce the recommended portion of any Brazil nuts to 3 nuts, to avoid Se overdosing [6].

The National Institutes of Health (NIH) in the U.S. established 2 terms with regards to nutrient consumption: 1. recommended dietary allowance (RDA)
- a value required to achieve optimal glutathione peroxidase (GPx) expression and in practice used to plan an adequate diet for healthy people, and 2. Upper limit (UL) - above which side effects were noted. In the case of Se, the recommended RDA-UL values from birth to 6 months of age is 15-45 $\mu \mathrm{g}$ /day (from breast milk), 6 months to13 years of age ranging from 20-45 to 40-280/day, and from 14 years until adulthood - 55-400 $\mu \mathrm{g} /$ day. The RDA is temporarily higher in pregnancy ( $60 \mu \mathrm{g} /$ day) and during lactation (70 $\mu \mathrm{g} /$ day) due to increased foetus and newborn requirements [7]. Similarly, the European Food Safety Authority (EFSA) recommended a higher (70 $\mu \mathrm{g} /$ day) adequate Se intake for adults (including pregnancy) and in lactating women (85 $\mu \mathrm{g} /$ day) [8]. The value for adults $(55 \mu \mathrm{g})$ is in accordance with the Scientific Committee for Food (SCF) of the European Commission, but the UL was set at a lower value of $300 \mu \mathrm{g} /$ day. However, the Reference Nutrient Intake (RNI) for Se in the UK is $60 \mu \mathrm{g} /$ day in women and $75 \mu \mathrm{g} /$ day in men and lactating women [9]. Nevertheless, most European countries have a Se daily intake lower than preferable (30-90 $\mu \mathrm{g} /$ day) [10]. As a result, some countries are Se-repleted, including North America, Venezuela, Greenland, Japan, and certain parts of China, whilst others are Se-depleted - the majority of countries in Europe, the Middle East, and certain parts of China [4]. Most European countries, except parts of Norway and Finland, have insufficient natural Se supplies, with the lowest values recorded in Eastern Europe. For example, Polish students have a deficient Se intake, with daily dose average around $25 \mu \mathrm{g} /$ day according to one study [11]. In the last decades, progressively lower Se concentrations have been reported in Polish women, regardless of their pregnancy status, probably due to decreased food Se intake. Moreover, in Polish pregnant females, Se serum status was lower than the European average $(31 \%$ had Se level < $50 \mu \mathrm{g} / \mathrm{L} v s .4 .5 \%$ in other European countries) [12]. A multicentre comparison between Italy, Greece, Romania, and Austria showed suboptimal Se levels in each cohort, with the highest serum Se concentrations in Italy [13]. Se status has also declined in the UK in the last decades, which coincides with the bread-making industry switching from high-Se wheat imported from North America to low-Se wheat grown in Europe or the UK, using fertilizers rich in S instead of Se, decreasing use of coal (Se-rich rock) as a fuel, and an increasing preference of a vegetarian diet over meat consumption [3]. An interesting example is northern Germany, where the division of countries with moderate Se in the west and those with low Se in the east follows the instated West-East German border. Such differences may be explained by the use of fertilisers with different Se content. The national program of implementing 
sodium selenate-rich fertilisers in primarily Se-depleted Finland in the early 1980s resulted in improvement of Se intake in the Finnish population from $30 \mathrm{mg} / \mathrm{d}$ to $100 \mathrm{mg} / \mathrm{d}$ in the late 1980s and promoted Finland to the list of European countries with adequate Se intake [14]

\section{Se status assessment}

Assessment of blood Se concentration may be misleading due to unspecified substitutions of SeMet for methionine in many proteins, where it is functionally inactive. Functional and individual Se status assessment may be evaluated using 2 markers, the activity of which is related to Se body supply: GPx concentration in whole blood, plasma, erythrocytes or thrombocytes and Selenoprotein P (SePP) in plasma/serum, where the latter is more accurate due to the crucial role in tissue Se distribution and higher required plasma Se concentration (100-120 $\mu \mathrm{g} / \mathrm{L})$ than for GPx $(90 \mu \mathrm{g} / \mathrm{L})$ to be fully expressed. Therefore, the optimal plasma/serum Se concentration of $120-125 \mu \mathrm{g} / \mathrm{L}$ reflects the maximal selenoprotein activity and sufficient Se storage. The positive correlation between blood Se and SePP exists in subjects until adequate Se concentration fully saturates the selenoprotein. However, both serum biomarkers are not tissue-specific and do not reflect the thyroid Se concentration $[8,15,16]$. Of note, there are no proposed region-specific reference ranges for plasma Se, which would be especially beneficial in the context of thyroid diseases, and no reliable thyroid biomarkers for Se status assessment are currently available.

\section{Se optimal supply and health}

According to Rayman et al., only Se-deficient individuals are reasonable candidates for substitution of this micronutrient due to the proposed U-shaped relationship between Se and health effects, where neither Se deficiency nor Se overdosing are beneficial [17].

Except for AIT, selenopenia is associated with increased risk for Keshan and Kashin-Beck disease, colorectal cancer in females and prostate cancer in males, infertility/reproduction complications, mental decline, more flawed immune defence, increased viral virulence, and higher mortality. Conversely, excessive Se intake produces selenosis (nail and hair loss, garlic breath odour, tachycardia, gastrointestinal and neurological symptoms), dermatitis, non-melanoma skin cancer, and increased prostate cancer risk and mortality. According to some studies, prolonged Se supplementation may also facilitate diabetes mellitus type 2 (DM2) development $[18,19]$. Nevertheless, the latest meta-analysis from 3 RCTs (SELECT, NPC, and Selenium Trial) analysing 20,290 participants with $200 \mu \mathrm{g}$ Se daily intake for 3-7.7 years, showed only a nonsignificant increase in DM2 occurrence between the study and placebo group (OR $=1.18,95 \%$ CI: 0.95-1.4). However, observational studies have linked high Se status with increased DM2 incidence $(\mathrm{OR}=2.03,95 \%$ CI: $1.51-2.72)$ [20]. Of note, the tendency was observed only in the highest Se concentration quartiles, in which sufficient Se supply was exceeded, and it mainly focussed on males [21]. On the other hand, DM2 or insulin resistance may trigger the liver to overproduce SePP, which alters per se plasma Se concentration. To explain whether high Se is a cause or effect of impaired glucose metabolism, convincing large RCTs and meta-analyses are needed [22]. Some authors associate Se deficiency with development of metabolic syndrome, obesity, and DM2 as a first-line consequence [21]

\section{Se in diet supplements}

The bioavailability, bioactivity, and potential toxicity of Se in the diet largely depend on the type of food and chemical properties of associated micronutrients. Se is present in the diet primarily in the organic form of selenocysteine (SeCys) and SeMet, which is most likely absorbed via transcellular diffusion in the small intestine [23]. On the other hand, the inorganic form of Se, selenite or selenate, is present in food to a lesser degree. SeMet is a form of Se that is most readily absorbed. At the same time, SeCys, selenate, and selenite are less bioavailable [24]. Supplementation of Se is frequently achieved using SeMet or Se yeast $(60 \%$ of which is SeMet) [25]. If supplementation is recommended to a patient, preference should be given to the organic form, SeMet, rather than inorganic. An attractive therapeutic option may be the patented Danish product by Pharma Nord (SelenoPrecise $\left.{ }^{\circledR}\right)$, used in large RCT (UK, Danish, Swedish PRECISE), which contains organic Se yeasts (100 $\mu$ g of Se per tablet, stable Se content: $66 \%$ of SeMet and $<1 \%$ of inorganic forms) with high bioavailability of $88.7 \%$, proven by the EFSA [26].

\section{Se supplementation in euthyroid AIT patients}

The idea of Se supplementation in thyroid diseases (mainly AIT) arose from epidemiological studies, in which Se-depleted regions had a higher number of AIT patients. A Chinese study of 3038 people from Se-rich and 3114 subjects from low-Se provinces proved increased prevalence and higher odds ratio (OR) for AIT $(\mathrm{OR}=0.47,95 \%$ CI: 0.35-0.65), SHT (OR $=0.68,95 \%$ CI: 0.58-0.93), overt hypothyroidism (OR $=0.75,95 \%$ CI: 0.63-0.90), and goitre (OR $=0.75,95 \%$ CI: 0.59-0.97) in the latter group ; altogether 18 vs. $30.5 \%$ ( $<<0.001$ ) 
[27]. In addition, in a comparator multi-centre study from 4 European low Se-equipped countries, the Se concentrations were significantly lower in AIT patients than in patients with non-AIT thyroid diseases [13]. The obtained results suggest that Se supplementation might reduce the incidence of thyroid diseases. The majority of studies concerning Se supplementation were performed on euthyroid AIT patients. The commonly measured primary outcome in those studies was a decrease in TA. However, some authors underlined the need to find clinical evidence, expressed by thyrotropin (TSH) decrease (or no progression to overt disease) or lowering LT4 dose in the course of combined LT4 and Se treatment. US echogenicity, oxidative and inflammatory body status, or QoL are frequently considered as secondary outcomes. Assessment of TA (the higher the level, the better the response), individual Se level at baseline (substitution or supplementation), geographical area of living, and iodine status (iodine deficiency exacerbates the effects of Se deficiency) should be taken into account in the interpretation of results. In the latest published meta-analysis (2017) concerning data from European and Brazilian patients, the authors failed to find the disease progression (expressed by TSH rise) in LT4-untreated subjects after 3, 6, or 12 months of Se supplementation [28]. Another meta-analysis (2016) by the same authors revealed that levels of anti-thyroid peroxidase antibodies (a-TPO) and anti-thyroglobulin antibodies (a-TG) declined significantly after 3 months in the group of patients who did not receive LT4. In the LT4-treated group a-TPO decreased at 3, 6, and 12 months, while a-TG decreased only after 12 months, which is in accordance with the previous meta-analysis results. Se supplementation favourably impacted the mood and general well-being of participants in this study [29, 30]. The countries that were analysed in these studies had a baseline plasma Se concentration below the normal range and reached a Se serum concentration of $70-85 \mu \mathrm{g} / \mathrm{L}$ and $37 \mu \mathrm{g} / \mathrm{L}$ upon conclusion of the European and Brazilian studies, respectively. Both meta-analyses underline no justification for routine Se supplementation in AIT euthyroid patients, due to lack of clinical evidence for improvement of the disease course. The adequately Se supplied AIT population without LT4 treatment (basal serum Se $110 \pm 16 \mu \mathrm{g} / \mathrm{L}$ in the study group and $123 \pm 19$ in healthy controls) had significantly decreased a-TPO, but not a-TG and $\mathrm{TSH}$, after 3 months of $200 \mu \mathrm{g} /$ day Se treatment in comparison to healthy controls. Additionally, the oxidative marker panel (total antioxidant capacity, superoxide dismutase, malondialdehyde) indicated an oxidative stress pattern in AIT patients compared to healthy controls. This improved significantly after Se intervention in the first group. The authors concluded that Se alleviates the thyroid autoimmune process by improving body antioxidative status [31]. Se supplementation (60 and $180 \mu \mathrm{g} /$ day) significantly reduced proinflammatory cytokine release mediated by INF- $\gamma$ (CXCL-9 and 10) in AIT euthyroid females who were not treated with LT4 in comparison to the placebo group. This serves as evidence for a Se-positive immunomodulatory effect and may prove advantageous in cases of an undetected change in thyroid parameters [32]. An Italian group (2016) found fT3 increases at 3 and 6 months and an fT4 decrease 3 months after Se supplementation in patients at the time of AIT diagnosis without LT4 treatment. The results of peripheral hormones were explained by the enhanced iodothyronine deiodinase activity due to incorporation of Se, which led to the augmented conversion of fT4 to fT3 [33]. Although Se supplementation resulted in a 9.9\% a-TPO decrease after 6 months in AIT patients in one study, its discontinuation led to an a-TPO level rise of $4.8 \%$ at 12 months. In contrast, the continuation of Se supplementation reduced a-TPO further by up to $21 \%$ at the above-mentioned time point. Interestingly, the observed a-TPO level in the group who continued the treatment at the end of the study was more reduced among non-smokers [34]. Nevertheless, no effect on a-TPO and TSH was seen in a well-designed, randomised, placebo-controlled, and double-blind study from an iodine-sufficient region, after $200 \mu \mathrm{g} /$ day sodium selenite for 6 months given to Se-deficient (not fully expressed SePP) euthyroid AIT females without LT4 treatment. The possible explanation may be that iodine-sufficient subjects are less sensitive to immunosuppressive Se properties [35]. Interindividual variations in response to Se supplementation among AIT patients may be explained by genetic variation. Genotyping revealed that single nucleotide polymorphism in r25191g/a of SePP gene (SEPP1) is expressed in Se responders, which may be associated with a more prominent a-TPO decline [36].

\section{Se supplementation in hypothyroid AIT patients}

The results of the "SETI" study were published in 2020. The research included patients with SHT, in whom TSH was within the range $4.26-10.00 \mathrm{mIU} / \mathrm{L}$, due to AIT (positive a-TPO), with no history of LT4 treatment. After 4 months of treatment with $83 \mu \mathrm{g} /$ day of SeMet, $48.9 \%$ of participants (responders) re-established euthyroidism (TSH $\leq 4.2)$; the rest (non-responders) remained hypothyroid (TSH > 4.2). At the end of the study, Se levels in serum significantly increased, while fT4 concentrations significantly decreased in 
both groups versus baseline. Six months after Se withdrawal, the TSH level was controlled again; in the non-responder group $14.2 \%$ of patients achieved euthyroidism, while in the responder group this was up to $83.3 \%$. The authors indicated that 4 months of Se supplementation allows for normalisation of TSH levels. Thus, Se is a promising therapeutic option for patients with SHT [37]. The aforementioned observation is pursuant with a previous study (2016), which was conducted on a group of patients with mild SHT due to AIT. The time of treatment and the dose of SeMet were the same. At the end of the study, $17.2 \%$ of participants were again euthyroid. Follow-up studies conducted 5 months later showed that euthyroidism was maintained mainly in the Se group [38]. These studies included patients with no history of thyroid hormone therapy, but Se treatment may also be useful as an adjunct to LT4 for patients with AIT and SHT. SeMet supplementation with $200 \mu \mathrm{g}$ /daily with LT4 for 6 months caused a significant reduction in a-TPO concentration: $46 \%$ after 3 months and $55.5 \%$ after 6 months. The group receiving only LT4 had a decrease of a-TPO of $21 \%$ after 3 months and $27 \%$ after 6 months. There were no significant changes in a-TG and thyroid hormone levels. The reduction of a-TPO levels might be the result of the influence of Se on the immune system and oxidative stress [39]. Se supplementation with LT4 is also effective in reducing the a-TPO level in AIT hypothyroid patients. Three months of treatment with $200 \mu \mathrm{g}$ sodium selenite/day + LT4 resulted in a significant reduction in a-TPO and a-TG levels [40]. Se added to LT4 in euthyroid and hypothyroid AIT patients decreased a-TPO and a-TG more than in patients treated exclusively with LT4. Moreover, euthyroid AIT patients were less Se deficient at baseline than hypothyroid ones, and the latter subgroup reached lower TA levels after Se treatment. These results showed that Se supplementation in addition to LT4 may be beneficial in the later disease stage. Additionally, Se intake significantly ameliorated levels of proinflammatory cytokine (IL-2) and therefore corrected disturbed immune balance in AIT patients, which was not observed in the group without Se supplementation [41]. However, another study showed that SeMet supplementation (200 $\mu \mathrm{g} /$ day) had no significant effect on the a-TPO level; however, this resulted in a substantial reduction in a-TG after 3 months of treatment [42]. The reason for the different responses to Se supplementation remains unclear. However, the duration of the treatment and the dose of Se preparations may affect the results. It is also possible that the initial concentration of TA, Se level, and thyroid disease duration play a crucial role in the outcome.

\section{Se supplementation in AIT and healthy pregnant women}

Se supplementation in pregnancy is currently not supported by the American Thyroid Association (ATA) due to conflicting results of studies, omitted impact of baseline Se and iodine status, and potential DM2 risk [43]. European Thyroid Association (ETA) guidelines do not raise the issue of Se supplementation for the management of subclinical hypothyroidism in pregnancy and children. Nevertheless, the majority of studies justify its potential advantages. Considering the physiological Se decline throughout pregnancy (partially due to haemodilution), the risk of developing severe Se deficiency is higher in this group [44]. In healthy pregnant females from Poland, Se concentrations physiologically decreased by $24 \%$ from the first trimester to the end of pregnancy [12]. However, Se deficits in AIT pregnant females were not more intensified than in healthy pregnant controls from a Se-depleted, mildly iodine-deficient area [45]. The question of whether low Se status has a negative impact on the course of AIT in pregnancy remains unanswered. In pregnant women, similarly to the general population, Se supplementation reduces the TA titre. Taking into account the narrow therapeutic index of Se, interventional studies on pregnant females are frequently characterized by lower Se proposed doses in comparison to the rest of the studied populations. The "SERENA" study (2019) demonstrated a significant reduction of a-TPO in the third trimester of pregnancy and 6 months after delivery in euthyroid females with AIT taking $83 \mu \mathrm{g}$ of SeMet since their first trimester. Although an a-TPO decrease during pregnancy is a natural immunosuppressive body reaction, preventing rejection of the foetus, TA tends to rise dramatically after delivery, as observed in the placebo group. The above-mentioned results provide evidence that Se supplementation during pregnancy reduces the risk of postpartum thyroiditis (PPT) [46]. This is in accordance with the previous finding, where $200 \mu \mathrm{g}$ of SeMet given to AIT women (with or without LT4) from the 10th gestation week until the postpartum period significantly reduced the incidence of PPT (28.6\% vs. $48.6 \%$ for placebo) and persistent hypothyroidism (11.7\% vs. 20.3\%). Additionally, the researchers observed an improvement in US echogenicity of thyroid parenchyma in the Se-treated group [47]. However, in euthyroid AIT patients with no additional LT4 treatment recruited for "SPRINT" (double-blind, randomized, placebo-controlled study), in which the group of 114 women from a mild-to-moderate iodine-deficient country received a lower dose of Se ( $60 \mu \mathrm{g} /$ day) from the $12^{\text {th }}$ week of pregnancy, no reduction in a-TPO (baseline lower median levels than 
in the previous study) was observed (54.2 vs. $65.6 \%$ in placebo). Nevertheless, Se modulated thyroid function by increasing fT4 $(p<0.029)$ and decreasing TSH $(p<0.050)$ throughout the pregnancy more in the Se group than in the placebo group. This may be explained by the additive Se immunomodulatory effect on the AIT course [48]. An interesting interventional clinical trial on the influence of immunomodulatory factors (including low Se dose) on intracytoplasmic sperm injection (ICSI) effectiveness in infertile AIT euthyroid patients was registered in 2019; however, the results have not been published yet [49].

Moreover, Se intake may also contribute to an uncomplicated pregnancy course. Within the "SPRINT" group, Se supplementation in Se-depleted subjects resulted in reduction of OR for developing pregnancy-induced hypertension or preeclampsia [50]. Women with the lowest Se serum concentration in the 10th-14th gestation week (from quartile $Q_{1}$ ) were 3 times more prone to deliver small-for-gestational-age (SGA) newborns $(\mathrm{OR}=3.02, \mathrm{p}=0.019)$ than in other quartiles [51]. Maternal Se-rich food intake was shown to decrease the risk of preterm birth [52]. In the population of women with spontaneous abortions, Se levels were significantly lower than in controls, potentially due to the associated risk of increased placental oxidative stress and trophoblast damage in early pregnancy [53].

Encouraging evidence originates from infertility studies of men and women, in which Se supplementation of $<200 \mu \mathrm{g} /$ day positively impacted sperm motility and enhanced oocyte cycle evolution, possibly by reducing oxidative stress [54].

Future double-blind placebo-controlled studies raising the issue of Se supplementation in pregnancy are warranted, especially in high-risk pregnancies in women from Se-depleted regions. Concomitant assessment of SePP as a marker of individual response for adjusting correct $\mathrm{Se}$ doses and the mutual interplay between different micronutrients may be relevant $[55,56]$.

\section{The impact of Se on quality of life}

Few clinical trials have assessed the effect of Se supplementation on QoL. According to these studies, Se supplementation (as sodium selenite or SeMet 200 $\mu \mathrm{g} /$ day for 3-6 months) resulted in improved well-being and mood and diminished fatigue in a group of patients with AIT, compared to controls $[39,57,58]$. More insight is expected from the "CATALYST" study, which is scheduled to be completed in December 2021. The aim of the study is to assess whether $200 \mu \mathrm{g} /$ day Se supplementation for 12 months alongside LT4 will have an impact on QoL in AIT patients [59].

\section{The impact of Se on the US image}

Se influence on thyroid US remains questionable. Supplementation of $50 \mu \mathrm{g}$ SeMet per day for 3 months among children with AIT led to thyroid volume regression by over 30\% [60]. In a study of a large group of patients (1100 women; 792 men), an inverse association between Se level and thyroid volume was shown $(p=0.003)$. In a group of tested women, an increase in serum Se concentration by $1 \mu \mathrm{mol} / \mathrm{L}$ caused a decrease in the thyroid gland volume by $0.18 \mathrm{~mL}$. Moreover, low Se status was significantly related to goitre risk (OR $0.07,95 \%$ CI: 0.008-0.6) and thyroid hypoechogenicity $(\mathrm{OR}=0.2,95 \%$ CI: 0.06-0.7), but not to thyroid nodules. In the male group, there was no relationship between Se level and thyroid structure or thyroid volume [61]. Another study showed that low Se concentration increases the risk of developing multiple nodules in the thyroid gland $(\mathrm{p}=0.087)$ [62]. A Se dose of $200 \mu \mathrm{g} /$ day with LT4 for 3 months in AIT patients improved thyroid US echogenicity [58]. On the other hand, in studies where Se was supplemented in a dose of $80 \mu \mathrm{g}, 160$ $\mu \mathrm{g}$, or $200 \mu \mathrm{g}$ for 3-12 months there was no change in echogenicity and volume of the thyroid gland at the end $[32,63]$. Further research is essential to find the optimal dose and time required for Se to exert its beneficial effects. It is necessary to investigate patterns of thyroid US in patients' who would benefit most from the supplementation.

\section{Se supplementation in non-AIT hypothyroidism}

Dietary Se deficiency may cause hypothyroidism, which was identified in 3 cases of children with SHT and no signs of infection. The thyroid US picture was unaffected. The plasma Se concentrations in subjects were 40 $\mu \mathrm{g} / \mathrm{L}, 32 \mu \mathrm{g} / \mathrm{L}$, and $43 \mu \mathrm{g} / \mathrm{L}$. Each child received $10 \mu \mathrm{g} / \mathrm{kg}$ daily of sodium selenite. After 4 weeks, clinical symptoms improved, and thyroid gland parameters and Se levels became normal. The authors concluded that the direct cause of hypothyroidism was Se deficiency and reduced activity of type II 5 '-deiodinase, which confirms the vital role of Se in the aetiology of hypothyroidism, even without concomitant AIT [64]. With that said, high-quality evidence is needed to confirm the above observation. This effect may be applicable to hypothyroidism coexisting with other end-stage chronic diseases, in which fT4 to fT3 conversion is impaired - for instance, in renal failure [65]. Determination of hypothyroid aetiology (AIT vs. non-AIT) and distinguishing these 2 subgroups would be advantageous in future clinical trials. 


\section{Se supplementation in a paediatric population}

A Se deficit in childhood is a considerable risk factor for poor physical and cognitive development [66]. Se plays an important role in synaptogenesis, myelination, and neuronal cell differentiation by regulating thyroid hormones [67]. Maternal erythrocyte Se (Ery-Se) concentrations were associated with neonatal development measures at 1.5 years [68]. An increase in maternal Se by $0.50 \mu \mathrm{g} / \mathrm{g}$ haemoglobin was associated with improved language comprehension by 3.7 points ( $0.5 \mathrm{SD}, 95 \%$ CI: $0.40-7.1, \mathrm{p}=0.028$ ) and increased psychomotor development by 12 points in girls (0.9 SD, 95\% CI: $4.3-19, \mathrm{p}=0.002)$ at 1.5 years of age. In a follow-up study, a positive association was also observed between maternal Ery-Se in early pregnancy and the outcomes in children at 10 years [69]. An increase of maternal Ery-Se from 5th to 95th percentile was associated with a full developmental score by approximately 8.1 points (95\% CI: 3.8-13). Similarly, a Polish study reported a significant positive association between Se levels in blood collected during the first trimester of pregnancy and motor skill development at 1-2 years of age and cognitive development at 2 years of age, and borderline association $(=0.2, \mathrm{p}=0.05)$ was observed for language development at 2 years of age [44]. A similar association between maternal urinary Se $(23 \pm 8.6$ $\mu \mathrm{g} / \mathrm{L})$ and cognition at 4 years of age was also reported in Greece $(n=575)$ [70]. Conversely, a recent study from Spain suggests an inversion of this relationship between maternal Se concentration and neuropsychological development at 1 year of age when the Se concentration in the first trimester exceeds $86 \mu \mathrm{g} / \mathrm{L}$ [71]. However, a study conducted in the U.S. did not identify an association between high levels of Ery-Se in pregnant women $(206 \mu \mathrm{g} / \mathrm{L}, \mathrm{n}=872)$ and children's cognitive function at 7.7 years of age [72]. Assessment of preschool children in Ethiopia revealed that low serum Se was associated with lower cognitive scores as validated tests [66]. Another body of research focuses on the effects of Se nutritional intake in children with autism spectrum disorder (ASD). A recent literature review of 4 studies found significant differences in Se tracing in the hair of children with ASD, compared to unaffected children [73]. Two of these 4 studies found increased Se levels in the hair of children with ASD, and the other 2 studies reported the opposite finding. With that said, more studies will be needed to evaluate the significance of Se and ASD association and its clinical implication. Additionally, Se deficiency was associated with the development of Keshan disease in children between the ages of 2 and 7 years and women of reproductive age. The possible aetiology may be related to mutation promoting in a poor Se environment in the genetic material of Coxsackie $\mathrm{B}$ virus's genetic material, resulting in its increased virulence and extensive myocardial damage leading to dilated cardiomyopathy [74]. An Se shortage in neonates and children is a risk factor for decreased motor and cognitive function. However, children's cognitive outcome is also affected by other factors associated, among others, with poor care, inadequate cognitive stimulation, and parasitic infections $[75,76]$.

\section{Se supplementation in adults and the elderly population}

Fluctuations in Se serum concentration are believed to intensify chronic inflammation, increase monocyte migration into the endothelium, and promote monocyte transformation into macrophages and eventually foam cells, thus advancing the development of atherosclerosis and increasing the risk of cardiovascular diseases (CVD) [77]. A recent meta-analysis reported a 50\% increase in Se concentration to be associated with $24 \%$ reduction in the risk of developing ischaemic heart disease [78]. A similar conclusion was drawn by a study that reported a reduced risk of CVD (RR: 0.66, 95\% CI: $0.40-1.09$ ) and mortality (RR: $0.69,95 \%$ CI: $0.57-0.84$ ) in individuals with high Se status [79]. Also, UK and Denmark PRECISE randomised, double-blinded, placebo-controlled, clinical trials explored the effects of Se supplementation on total cholesterol (T-C) and non-HDL cholesterol $[80,81]$. In the UK PRECISE clinical study ( $\mathrm{n}=501)$, healthy participants (60 to 74 years old) were supplemented with 100, 200, or $300 \mu \mathrm{g} /$ day Se-enriched yeast tablets or placebo for 6 months [80]. Compared to placebo, Se supplementation of 100 and $200 \mu \mathrm{g}$ /day significantly decreased T-C and non-HDL cholesterol in the plasma. Supplementation with 300 $\mu \mathrm{g} /$ day for 6 months significantly increased HDL-cholesterol but did not affect either total or non-HDL cholesterol. Conversely, a similar study carried out in a Danish population found no significant difference in total or HDL cholesterol concentrations between placebo and Se supplementation groups after 6 months or 5 years, despite similarities in Se baseline concentrations in the 2 populations $(88.6 \mu \mathrm{g} / \mathrm{L}$ in Denmark, $91.2 \mu \mathrm{g} / \mathrm{L}$ in the UK) [81]. Se homeostasis is also implicated in the maintenance of blood pressure. A recent observational study conducted in a group of 2169 Inuit described a potential blood pressure-lowering effect seen with an Se-rich diet [82]. Participants with low Se and high mercury serum levels were more likely to develop CVD, including hypertension $(\mathrm{OR}=1.76)$, stroke $(\mathrm{OR}=1.57)$, and heart attack $(O R=1.26)$. In contrast, other studies described a positive association between high Se 
serum concentration and hypertension $[83,84]$. Based on the given research, the cardiovascular benefits of Se supplementation are unclear and should not be recommended. Both Se deficiency and excess have been associated with increased mortality. According to U.S. Third National Health and Nutrition Examination survey analysis $(\mathrm{n}=13887)$, mortality exhibited a U-shaped association with the lowest mortality rate at a serum Se concentration of $135 \mu \mathrm{g} / \mathrm{L}$ and high mortality with either lower or higher Se concentration [4, 85]. In the Danish PRECISE clinical trial, mortality was assessed 10 years after 5-year supplementation with 100,200 , or $300 \mu \mathrm{g}$ Se/day as Se-enriched yeast or placebo yeast [86]. Supplementation with $300 \mu \mathrm{g}$ Se/day resulted in an $11.3 \%$ (95\% CI: $0.0-22.6 \%$ ) increase in all-cause mortality compared to placebo 10 years after treatment. Conversely, a prospective study found an inverse relationship between Se intake and all-cause CVD mortality in men and women. However, no significant difference in mortality was observed between Se intake and cancer-related mortality in both sexes [87].

\section{Conclusions}

According to our literature review, the recommendation of Se supplementation is supported by everyday clinical practice. However, Se supplement seems to be uncontrollably prescribed (no baseline and follow-up Se assessment) and not adjusted to the specific population's profile (children, adults, males, females, pregnant, non-pregnant, AIT, non-AIT thyroid disease, and area of living). According to the authors' best knowledge, no commercially available oral Se preparation has been approved by the U.S. Food and Drug Administration. Thus, available Se formulations and effective doses need to be revised and standardised. The reference ranges for Se concentrations in various geographical areas are lacking, and there is a need to determine thyroid tissue-specific biomarkers for Se functional assessment. Clinical trials have explored many health benefits of Se supplementation; however, its narrow therapeutic index, patients' geographical location, individual Se intake, and specific clinical setting should be considered to evaluate the benefit-risk balance before Se supplementation. Future studies comparing regions with varying natural supplies of Se and iodine and investigating the effects of the interplay of these elements on the course of AIT would be valuable. Among successful Se supplementation candidates may be pregnant females, especially those from iodine- and Se-depleted areas, with risk factors like AIT. Se implementation in this population may prevent the development of selenopenia, PPT, hypertension, and poor neonatal outcomes. However, clear recommendations on indi- cations for treatment, therapy duration, and suitable doses need to be established. Factors determining the response to Se should be elucidated, taking into consideration genetic predisposition and mutual interrelations with other trace elements.

\section{References}

1. Negro R, Attanasio R, Grimaldi F, et al. A 2016 Italian Survey about the Clinical Use of Selenium in Thyroid Disease. Eur Thyroid J. 2016; 5(3): 164-170, doi: 10.1159/000447667, indexed in Pubmed: 27843806.

2. Winther KH, Papini E, Attanasio R, et al. A 2018 European Thyroid Association Survey on the Use of Selenium Supplementation in Hashimoto's Thyroiditis. Eur Thyroid J. 2020; 9(2): 99-105, doi: 10.1159/000504781, indexed in Pubmed: 32257959.

3. Johnson CC, Fordyce FM, Rayman MP. Symposium on 'Geographical and geological influences on nutrition': Factors controlling the distribution of selenium in the environment and their impact on health and nutrition. Proc Nutr Soc. 2010; 69(1): 119-132, doi: 10.1017/S0029665109991807, indexed in Pubmed: 19968907.

4. Rayman M. Selenium and human health. Lancet. 2012; 379(9822): 1256-1268, doi: 10.1016/s0140-6736(11)61452-9, indexed in Pubmed: 22381456.

5. Office of Dietary Supplements — Selenium. https://ods.od.nih.gov/factsheets/Selenium-HealthProfessional/ (January 22, 2021).

6. Lima LW, Stonehouse GC, Walters C, et al. Selenium Accumulation, Speciation and Localization in Brazil Nuts (H.B.K.). Plants (Basel). 2019; 8(8), doi: 10.3390/plants8080289, indexed in Pubmed: 31426292.

7. Institute of Medicine (US) Panel on Dietary Antioxidants and Related Compounds. Dietary Reference Intakes for Vitamin C, Vitamin E, Selenium, and Carotenoids. National Academies Press (US); 2000. http:/ www.ncbi.nlm.nih.gov/books/NBK225483/ (January 24, 2021.).

8. Scientific Opinion on Dietary Reference Values for selenium. EFSA J. 2014; 12(10): 3846, doi: 10.2903/j.efsa.2014.3846.

9. Dietary reference values for food energy and nutrients for the United Kingdom. Report of the Panel on Dietary Reference Values of the Committee on Medical Aspects of Food Policy. Rep Health Soc Subj (Lond). 1991; 41: 1-210, indexed in Pubmed: 1961974.

10. Proposed nutrient and energy intakes for the European community: a report of the Scientific Committee for Food of the European community. Nutr Rev. 1993; 51(7): 209-212, doi: 10.1111/j.1753-4887.1993. tb03106.x, indexed in Pubmed: 8414226.

11. Skibniewska KA, Dymkowska-Malesa M, Siwik P, et al. [Nutritive value of Olsztyn University students diet]. Przegl Lek. 2007; 64 Suppl 4: 15-18, indexed in Pubmed: 18543423.

12. Wasowicz W, Gromadzinska J, Rydzynski K, et al. Selenium status of low-selenium area residents: Polish experience. Toxicol Lett. 2003; 137(1-2): 95-101, doi: 10.1016/s0378-4274(02)00383-1, indexed in Pubmed: 12505435 .

13. Krassas GE, Pontikides N, Tziomalos K, et al. Selenium status in patients with autoimmune and non-autoimmune thyroid diseases from four European countries. Expert Rev Endocrinol Metab. 2014; 9(6): 685-692, doi: 10.1586/17446651.2014.960845, indexed in Pubmed: 30736204.

14. Varo P, Alfthan G, Ekholm P, et al. Selenium intake and serum selenium in Finland: effects of soil fertilization with selenium. Am J Clin Nutr. 1988; 48(2): 324-329, doi: 10.1093/ajcn/48.2.324, indexed in Pubmed: 2841842.

15. Kipp AP, Strohm D, Brigelius-Flohé R, et al. German Nutrition Society (DGE). Revised reference values for selenium intake. J Trace Elem Med Biol. 2015; 32: 195-199, doi: 10.1016/j.jtemb.2015.07.005, indexed in Pubmed: 26302929.

16. Köhrle J. Selenium and the thyroid. Curr Opin Endocrinol Diabetes Obes. 2015; 22(5): 392-401, doi: 10.1097/MED.0000000000000190, indexed in Pubmed: 26313901.

17. Winther KH, Rayman MP, Bonnema SJ, et al. Selenium in thyroid disorders - essential knowledge for clinicians. Nat Rev Endocrinol. 2020; 16(3): 165-176, doi: 10.1038/s41574-019-0311-6, indexed in Pubmed: 32001830.

18. Stranges S, Marshall JR, Natarajan R, et al. Effects of long-term selenium supplementation on the incidence of type 2 diabetes: a randomized trial. Ann Intern Med. 2007; 147(4): 217-223, doi: 10.7326/0003-4819-147-4-20 0708210-00175, indexed in Pubmed: 17620655.

19. Kohler LN, Florea A, Kelley CP, et al. Higher Plasma Selenium Concentrations Are Associated with Increased Odds of Prevalent Type 2 Diabetes. J Nutr. 2018; 148(8): 1333-1340, doi: 10.1093/jn/nxy099, indexed in Pubmed: 29924331.

20. Kohler LN, Foote J, Kelley CP, et al. Selenium and Type 2 Diabetes: Systematic Review. Nutrients. 2018; 10(12), doi: 10.3390/nu10121924, indexed in Pubmed: 30563119

21. Schomburg L. The other view: the trace element selenium as a micronutrient in thyroid disease, diabetes, and beyond. Hormones (Ath- 
ens). 2020; 19(1): 15-24, doi: 10.1007/s42000-019-00150-4, indexed in Pubmed: 31823341.

22. Mao J, Teng $\mathrm{W}$. The relationship between selenoprotein $\mathrm{P}$ and glucose metabolism in experimental studies. Nutrients. 2013; 5(6): 1937-1948, doi: 10.3390/nu5061937, indexed in Pubmed: 23760059.

23. Thiry C, Ruttens A, Pussemier L, et al. An in vitro investigation of species-dependent intestinal transport of selenium and the impact of this process on selenium bioavailability. Br J Nutr. 2013; 109(12): 2126-2134, doi: 10.1017/S0007114512004412, indexed in Pubmed: 23148951.

24. Kumar A, Prasad KS. Role of nano-selenium in health and environment. J Biotechnol. 2021; 325: 152-163, doi: 10.1016/j.jbiotec.2020.11.004, indexed in Pubmed: 33157197.

25. Rayman MP. The use of high-selenium yeast to raise selenium status: how does it measure up? Br J Nutr. 2004; 92(4): 557-573, doi: 10.1079/bjn20041251, indexed in Pubmed: 15522125.

26. Bügel S, Larsen EH, Sloth JJ, et al. Absorption, excretion, and retention of selenium from a high selenium yeast in men with a high intake of selenium. Food Nutr Res. 2008; 52, doi: 10.3402/fnr.v52i0.1642, indexed in Pubmed: 19109661.

27. Wu Q, Rayman MP, Lv H, et al. Low Population Selenium Status Is Associated With Increased Prevalence of Thyroid Disease. J Clin Endocrinol Metab. 2015; 100(11): 4037-4047, doi: 10.1210/jc.2015-2222, indexed in Pubmed: 26305620.

28. Winther $\mathrm{KH}$, Wichman JE, Bonnema SJ, et al. Insufficient documentation for clinical efficacy of selenium supplementation in chronic autoimmune thyroiditis, based on a systematic review and meta-analysis. Endocrine. 2017; 55(2): 376-385, doi: 10.1007/s12020-016-1098-z, indexed in Pubmed: 27683225.

29. Wichman J, Winther KH, Bonnema SJ, et al. Selenium Supplementation Significantly Reduces Thyroid Autoantibody Levels in Patients with Chronic Autoimmune Thyroiditis: A Systematic Review and Meta-Analysis. Thyroid. 2016; 26(12): 1681-1692, doi: 10.1089/thy.2016.0256, indexed in Pubmed: 27702392.

30. Fan $\mathrm{Y}, \mathrm{Xu} \mathrm{S}$, Zhang H, et al. Selenium supplementation for autoimmune thyroiditis: a systematic review and meta-analysis. Int J Endocrinol. 2014; 2014: 904573, doi: 10.1155/2014/904573, indexed in Pubmed: 25574167.

31. Tian X, Li N, Su R, et al. Selenium Supplementation May Decrease Thyroid Peroxidase Antibody Titer via Reducing Oxidative Stress in Euthyroid Patients with Autoimmune Thyroiditis. Int J Endocrinol. 2020; 2020: 9210572, doi: 10.1155/2020/9210572, indexed in Pubmed: 32676110

32. Pilli T, Cantara S, Schomburg L, et al. IFN $\gamma$-Inducible Chemokines Decrease upon Selenomethionine Supplementation in Women with Euthyroid Autoimmune Thyroiditis: Comparison between Two Doses of Selenomethionine ( 80 or $160 \mu \mathrm{g}$ ) versus Placebo. Eur Thyroid J. 2015; 4(4): 226-233, doi: 10.1159/000439589, indexed in Pubmed: 26835425.

33. Esposito D, Rotondi M, Accardo G, et al. Influence of short-term selenium supplementation on the natural course of Hashimoto's thyroiditis: clinical results of a blinded placebo-controlled randomized prospective trial. J Endocrinol Invest. 2017; 40(1): 83-89, doi: 10.1007/s40618-016-0535-4, indexed in Pubmed: 27572248.

34. Mazokopakis EE, Papadakis JA, Papadomanolaki MG, et al. Effects of 12 months treatment with L-selenomethionine on serum anti-TPO Levels in Patients with Hashimoto's thyroiditis. Thyroid. 2007; 17(7): 609-612, doi: 10.1089/thy.2007.0040, indexed in Pubmed: 17696828.

35. Eskes SA, Endert E, Fliers E, et al. Selenite supplementation in euthyroid subjects with thyroid peroxidase antibodies. Clin Endocrinol (Oxf). 2014; 80(3): 444-451, doi: 10.1111/cen.12284, indexed in Pubmed: 23844613.

36. Wang W, Mao J, Zhao J, et al. Decreased Thyroid Peroxidase Antibody Titer in Response to Selenium Supplementation in Autoimmune Thyroiditis and the Influence of a Selenoprotein P Gene Polymorphism: A Prospective, Multicenter Study in China. Thyroid. 2018; 28(12): 1674-1681, doi: 10.1089/thy.2017.0230, indexed in Pubmed: 30398407.

37. Pirola I, Rotondi M, Cristiano A, et al. Selenium supplementation in patients with subclinical hypothyroidism affected by autoimmune thyroiditis: Results of the SETI study. Endocrinol Diabetes Nutr. 2020;67(1): 28-35, doi: 10.1016/j.endinu.2019.03.018, indexed in Pubmed: 31196739.

38. Pirola I, Gandossi E, Agosti B, et al. Selenium supplementation could restore euthyroidism in subclinical hypothyroid patients with autoimmune thyroiditis. Endokrynol Pol. 2016; 67(6): 567-571, doi: 10.5603/EP.2016.0064, indexed in Pubmed: 28042649.

39. Duntas LH, Mantzou E, Koutras DA. Effects of a six month treatment with selenomethionine in patients with autoimmune thyroiditis. Eur J Endocrinol. 2003; 148(4): 389-393, doi: 10.1530/eje.0.1480389, indexed in Pubmed: 12656658.

40. Kachouei A, Rezvanian H, Amini M, et al. The Effect of Levothyroxine and Selenium versus Levothyroxine Alone on Reducing the Level of Anti-thyroid Peroxidase Antibody in Autoimmune Hypothyroid Patients. Adv Biomed Res. 2018; 7: 1, doi: 10.4103/2277-9175.223735, indexed in Pubmed: 29456972

41. Yu L, Zhou L, Xu E, et al. Levothyroxine monotherapy versus levothyroxine and selenium combination therapy in chronic lym- phocytic thyroiditis. J Endocrinol Invest. 2017; 40(11): 1243-1250, doi: 10.1007/s40618-017-0693-z, indexed in Pubmed: 28534148.

42. Anastasilakis AD, Toulis KA, Nisianakis P, et al. Selenomethionine treatment in patients with autoimmune thyroiditis: a prospective, quasi-randomised trial. Int J Clin Pract. 2012; 66(4): 378-383, doi: 10.111 1/j.1742-1241.2011.02879.x, indexed in Pubmed: 22356267.

43. Alexander EK, Pearce EN, Brent GA, et al. 2017 Guidelines of the American Thyroid Association for the Diagnosis and Management of Thyroid Disease During Pregnancy and the Postpartum. Thyroid. 2017; 27(3): 315-389, doi: 10.1089/thy.2016.0457, indexed in Pubmed: 28056690

44. Polanska K, Krol A, Sobala W, et al. Selenium status during pregnancy and child psychomotor development-Polish Mother and Child Cohort study. Pediatr Res. 2016; 79(6): 863-869, doi: 10.1038/pr.2016.32, indexed in Pubmed: 26885758.

45. Ambroziak U, Hybsier S, Shahnazaryan U, et al. Severe selenium deficits in pregnant women irrespective of autoimmune thyroid disease in an area with marginal selenium intake. J Trace Elem Med Biol. 2017; 44 186-191, doi: 10.1016/j.jtemb.2017.08.005, indexed in Pubmed: 28965575.

46. Mantovani G, Isidori AM, Moretti C, et al. Selenium supplementation in the management of thyroid autoimmunity during pregnancy: results of the "SERENA study", a randomized, double-blind, placebo-controlled trial. Endocrine. 2019; 66(3): 542-550, doi: 10.1007/s12020-019-01958-1, indexed in Pubmed: 31129812.

47. Negro R, Greco G, Mangieri T, et al. The influence of selenium supplementation on postpartum thyroid status in pregnant women with thyroid peroxidase autoantibodies. J Clin Endocrinol Metab. 2007; 92(4): 1263-1268, doi: 10.1210/jc.2006-1821, indexed in Pubmed: 17284630

48. Mao J, Pop VJ, Bath SC, et al. Effect of low-dose selenium on thyroid autoimmunity and thyroid function in UK pregnant women with mild-to-moderate iodine deficiency. Eur J Nutr. 2016; 55(1): 55-61, doi: 10.1007/s00394-014-0822-9, indexed in Pubmed: 25524327.

49. Aljazeera Hospital. The Role of Immunomodulatory Treatment in Success of ICSI in Patients Who Have Infertility With Autoimmune Thyroiditis. clinicaltrials.gov; 2020. https://clinicaltrials. gov/ct2/show/NCT03289403 (January 18, 2021).

50. Rayman MP, Bath SC, Westaway J, et al. Selenium status in U.K. pregnant women and its relationship with hypertensive conditions of pregnancy. Br J Nutr. 2015; 113(2): 249-258, doi: 10.1017/S000711451400364X, indexed in Pubmed: 25571960

51. Lewandowska M, Sajdak S, Lubiński J. The Role of Early Pregnancy Maternal Selenium Levels on the Risk for Small-for-Gestational Age Newborns. Nutrients. 2019; 11(10), doi: 10.3390/nu11102298, indexed in Pubmed: 31561532

52. Barman M, Brantsæter AL, Nilsson S, et al. Maternal dietary selenium intake is associated with increased gestational length and decreased risk of preterm delivery. Br J Nutr. 2020; 123(2): 209-219, doi: 10.1017/S0007114519002113, indexed in Pubmed: 31865927.

53. Abdulah $\mathrm{R}$, Noerjasin $\mathrm{H}$, Septiani $\mathrm{L}$, et al. Reduced serum selenium concentration in miscarriage incidence of Indonesian subjects. Biol Trace Elem Res. 2013; 154(1): 1-6, doi: 10.1007/s12011-013-9701-0, indexed in Pubmed: 23695728

54. Mintziori G, Mousiolis A, Duntas LH, et al. Evidence for a manifold role of selenium in infertility. Hormones (Athens). 2020; 19(1): 55-59, doi: 10.1007/s42000-019-00140-6, indexed in Pubmed: 31701489.

55. Hubalewska-Dydejczyk A, Duntas L, Gilis-Januszewska A. Pregnancy, thyroid, and the potential use of selenium. Hormones (Athens). 2020; 19(1): 47-53, doi: 10.1007/s42000-019-00144-2, indexed in Pubmed: 31721136

56. Duntas LH. Selenium and at-risk pregnancy: challenges and controversies. Thyroid Res. 2020; 13: 16, doi: 10.1186/s13044-020-00090-x, indexed in Pubmed: 33014140.

57. Karanikas G, Schuetz M, Kontur S, et al. No immunological benefit of selenium in consecutive patients with autoimmune thyroiditis. Thyroid. 2008; 18(1): 7-12, doi: 10.1089/thy.2007.0127, indexed in Pubmed: 18302514.

58. Gärtner R, Gasnier BCH, Dietrich JW, et al. Selenium supplementation in patients with autoimmune thyroiditis decreases thyroid peroxidase antibodies concentrations. J Clin Endocrinol Metab. 2002; 87(4): 1687-1691, doi: 10.1210/jcem.87.4.8421, indexed in Pubmed: 11932302

59. Winther KH, Watt T, Bjørner JB, et al. The chronic autoimmune thyroiditis quality of life selenium trial (CATALYST): study protocol for a randomized controlled trial. Trials. 2014; 15: 115, doi: 10.1186/1745-6215-15-115, indexed in Pubmed: 24716668

60. Onal H, Keskindemirci G, Adal E, et al. Effects of selenium supplementation in the early stage of autoimmune thyroiditis in childhood: an open-label pilot study. J Pediatr Endocrinol Metab. 2012; 25(7-8): 639-644, doi: 10.1515/jpem-2012-0078, indexed in Pubmed: 23155687.

61. Derumeaux H, Valeix P, Castetbon $\mathrm{K}$, et al. Association of selenium with thyroid volume and echostructure in 35- to 60-year-old French adults. Eur J Endocrinol. 2003; 148(3): 309-315, doi: 10.1530/eje.0.1480309, indexed in Pubmed: 12611611 
62. Rasmussen LB, Schomburg L, Köhrle J, et al. Selenium status, thyroid volume, and multiple nodule formation in an area with mild iodine deficiency. Eur J Endocrinol. 2011; 164(4): 585-590, doi: 10.1530/EJE-10-1026, indexed in Pubmed: 21242171.

63. de Farias CR, Cardoso BR, de Oliveira GMB, et al. A randomized-controlled, double-blind study of the impact of selenium supplementation on thyroid autoimmunity and inflammation with focus on the GPx1 genotypes. J Endocrinol Invest. 2015; 38(10): 1065-1074, doi: 10.1007/s40618-015-0285-8, indexed in Pubmed: 25894865.

64. Pizzulli A, Ranjbar A. Selenium Deficiency and Hypothyroidism : A New Etiology in the Differential Diagnosis of Hypothyroidism in Children. Biol Trace Elem Res. 2000; 77(3): 199-208, doi: 10.1385/bter:77:3:199, indexed in Pubmed: 11204462.

65. Dubczak I, Niemczyk L, Szamotulska K, et al. The influence of hypothyroidism and substitution treatment on thyroid hormone conversion ratios and rT3 concentration in patients with end-stage renal failure. Endokrynol Pol. 2019; 70(2): 165-171, doi: 10.5603/EP.a2018.0087, indexed in Pubmed: 30480752

66. Gashu D, Stoecker BJ, Bougma K, et al. Stunting, selenium deficiency and anemia are associated with poor cognitive performance in preschool children from rural Ethiopia. Nutr J. 2016; 15: 38 , doi: 10.1186/s12937-016-0155-z, indexed in Pubmed: 27067274.

67. Sher L. Role of thyroid hormones in the effects of selenium on mood, behavior, and cognitive function. Med Hypotheses. 2001; 57(4): 480-483, doi: 10.1054/mehy.2001.1369, indexed in Pubmed: 11601874.

68. Skröder HM, Hamadani JD, Tofail F, et al. Selenium status in pregnancy influences children's cognitive function at 1.5 years of age. Clin Nutr. 2015; 34(5): 923-930, doi: 10.1016/j.clnu.2014.09.020, indexed in Pubmed: 25444556.

69. Skröder H, Kippler M, Tofail F, et al. Early-Life Selenium Status and Cognitive Function at 5 and 10 Years of Age in Bangladeshi Children. Environ Health Perspect. 2017; 125(11): 117003, doi: 10.1289/EHP1691, indexed in Pubmed: 29116931.

70. Kippler M, Bottai M, Georgiou V, et al. Impact of prenatal exposure to cadmium on cognitive development at preschool age and the importance of selenium and iodine. Eur J Epidemiol. 2016; 31(11): 1123-1134, doi: 10.1007/s10654-016-0151-9, indexed in Pubmed: 27147065.

71. Amorós R, Murcia M, Ballester F, et al. Selenium status during pregnancy: Influential factors and effects on neuropsychological development among Spanish infants. Sci Total Environ. 2018; 610-611: 741-749, doi: 10.1016/j.scitotenv.2017.08.042, indexed in Pubmed: 28822941.

72. Oken E, Rifas-Shiman SL, Amarasiriwardena C, et al. Maternal prenatal fish consumption and cognition in mid childhood: Mercury, fatty acids, and selenium. Neurotoxicol Teratol. 2016; 57: 71-78, doi: 10.1016/j. ntt.2016.07.001, indexed in Pubmed: 27381635

73. Tinkov AA, Skalnaya MG, Simashkova NV, et al. Association between catatonia and levels of hair and serum trace elements and minerals in autism spectrum disorder. Biomed Pharmacother. 2019; 109: 174-180, doi: 10.1016/j.biopha.2018.10.051, indexed in Pubmed: 30396074.

74. Lei C, Niu X, Ma X, et al. Is selenium deficiency really the cause of Keshan disease? Environ Geochem Health. 2011; 33(2): 183-188, doi: 10.1007/s10653-010-9331-9, indexed in Pubmed: 20574681.
75. Walker S, Wachs T, Gardner JM, et al. Child development: risk factors for adverse outcomes in developing countries. Lancet. 2007; 369(9556): 145-157, doi: 10.1016/s0140-6736(07)60076-2, indexed in Pubmed: 17223478 .

76. Berkman D, Lescano A, Gilman R, et al. Effects of stunting, diarrhoeal disease, and parasitic infection during infancy on cognition in late childhood: a follow-up study. Lancet. 2002; 359(9306): 564-571, doi: 10.1016/s0140-6736(02)07744-9, indexed in Pubmed: 11867110.

77. Gać P, Czerwińska K, Macek P, et al. The importance of selenium and zinc deficiency in cardiovascular disorders. Environ Toxicol Pharmacol. 2021; 82: 103553, doi: 10.1016/j.etap.2020.103553, indexed in Pubmed: 33238203.

78. Zhang X, Liu C, Guo J, et al. Selenium status and cardiovascular diseases: meta-analysis of prospective observational studies and randomized controlled trials. Eur J Clin Nutr. 2016; 70(2): 162-169, doi: 10.1038/ejcn.2015.78, indexed in Pubmed: 25990689.

79. Kuria A, Tian H, Li M, et al. Selenium status in the body and cardiovascular disease: a systematic review and meta-analysis. Crit Rev Food Sci Nutr. 2020 [Epub ahead of print]: 1-10, doi: 10.1080/10408398.2020.1803 200, indexed in Pubmed: 32799545.

80. Rayman MP, Stranges S, Griffin BA, et al. Effect of supplementation with high-selenium yeast on plasma lipids: a randomized trial. Ann Intern Med. 2011; 154(10): 656-665, doi: 10.7326/0003-4819-154-10-201105170-0 0005, indexed in Pubmed: 21576533.

81. Cold $\mathrm{F}$ Winther $\mathrm{KH}$, Pastor-Barriuso $\mathrm{R}$, et al Randomised controlled trial of the effect of long-term selenium supplementation on plasma cholesterol in an elderly Danish population. Br J Nutr. 2015; 114(11): 1807-1818, doi: 10.1017/S0007114515003499, indexed in Pubmed: 26420334.

82. $\mathrm{Hu} \mathrm{XF}$, Eccles KM, Chan HM. High selenium exposure lowers the odd ratios for hypertension, stroke, and myocardial infarction associated with mercury exposure among Inuit in Canada. Environ Int. 2017; 102: 200-206, doi: 10.1016/j.envint.2017.03.002, indexed in Pubmed: 28279481.

83. Bastola MM, Locatis C, Maisiak R, et al. Selenium, copper, zinc and hypertension: an analysis of the National Health and Nutrition Examination Survey (2011-2016). BMC Cardiovasc Disord. 2020; 20(1): 45, doi: 10.1186/s12872-020-01355-x, indexed in Pubmed: 32005161.

84. Vinceti M, Chawla R, Filippini T, et al. Blood pressure levels and hypertension prevalence in a high selenium environment: results from a cross-sectional study. Nutr Metab Cardiovasc Dis. 2019; 29(4): 398-408, doi: 10.1016/j.numecd.2019.01.004, indexed in Pubmed: 30782506.

85. Bleys J, Navas-Acien A, Guallar E. Serum selenium levels and all-cause, cancer, and cardiovascular mortality among US adults. Arch Intern Med. 2008; 168(4): 404-410, doi: 10.1001/archinternmed.2007.74, indexed in Pubmed: 18299496.

86. Rayman MP, Winther KH, Pastor-Barriuso R, et al. Effect of long-term selenium supplementation on mortality: Results from a multiple-dose, randomised controlled trial. Free Radic Biol Med. 2018; 127: 46-54, doi: 10.1016/j.freeradbiomed.2018.02.015, indexed in Pubmed: 29454039.

87. Sun JW, Shu XO, Li HL, et al. Dietary selenium intake and mortality in two population-based cohort studies of 133957 Chinese men and women. Public Health Nutr. 2016; 19(16): 2991-2998, doi: 10.1017/S1368980016001130, indexed in Pubmed: 27197889. 